\title{
HOLLOW QUASI-FATOU COMPONENTS OF QUASIREGULAR MAPS
}

\author{
DANIEL A. NICKS, DAVID J. SIXSMITH
}

\begin{abstract}
We define a quasi-Fatou component of a quasiregular map as a connected component of the complement of the Julia set. A domain in $\mathbb{R}^{d}$ is called hollow if it has a bounded complementary component. We show that for each $d \geq 2$ there exists a quasiregular map of transcendental type $f: \mathbb{R}^{d} \rightarrow \mathbb{R}^{d}$ with a quasi-Fatou component which is hollow.

Suppose that $U$ is a hollow quasi-Fatou component of a quasiregular map of transcendental type. We show that if $U$ is bounded, then $U$ has many properties in common with a multiply connected Fatou component of a transcendental entire function. On the other hand, we show that if $U$ is not bounded, then it is completely invariant and has no unbounded boundary components. We show that this situation occurs if $J(f)$ has an isolated point, or if $J(f)$ is not equal to the boundary of the fast escaping set. Finally, we deduce that if $J(f)$ has a bounded component, then all components of $J(f)$ are bounded.
\end{abstract}

\section{INTRODUCTION}

In the study of complex dynamics, the first example of a transcendental entire function with a multiply connected Fatou component was given by Baker [1] over half a century ago. We refer to the survey [4] for definitions and further background on complex dynamics. Since Baker's paper many authors have studied multiply connected Fatou components; see, for example, the papers [2, 3, 11, 23, 26].

In this paper we extend this study to more than two (real) dimensions, for the first time. Suppose that $d \geq 2$, and that $f: \mathbb{R}^{d} \rightarrow \mathbb{R}^{d}$ is a quasiregular map of transcendental type. We refer to Section 2 for a definition of a quasiregular map; intuitively, this is a map with a bounded amount of local distortion. A quasiregular map is said to be of transcendental type if it has an essential singularity at infinity. In this setting we need a different definition of the Julia set to that used in complex dynamics. Following [6] and [10], we define the Julia set $J(f)$ to be the set of all $x \in \mathbb{R}^{d}$ such that

$$
\operatorname{cap}\left(\mathbb{R}^{d} \backslash \bigcup_{k=1}^{\infty} f^{k}(U)\right)=0,
$$

for every neighbourhood $U$ of $x$. Here, if $S \subset \mathbb{R}^{d}$, then cap $S=0$ means that $S$ is, in a precise sense, a "small" set; we refer to Section 2 for a full definition. It is known [10, Theorem 1.1] that if $f$ is a quasiregular map of transcendental type, then card $(J(f))=\infty$.

2010 Mathematics Subject Classification. Primary 37F10; Secondary 30C65, 30D05.

Both authors were supported by Engineering and Physical Sciences Research Council grant $\mathrm{EP} / \mathrm{L} 019841 / 1$. 
We are not aware that any author has studied the complement of the Julia set of a general quasiregular map. We define the quasi-Fatou set $Q F(f)$ as the complement in $\mathbb{R}^{d}$ of the Julia set. Thus the quasi-Fatou set is an open set which, if non-empty, has the Julia set as its boundary. Observe that we make no assumptions about the normality of the family of iterates $\left(f^{k}\right)_{k \geq 0}$ in a quasi-Fatou component, which is a connected component of the quasi-Fatou set. A set $S$ is said to be completely invariant if $x \in S$ implies that $f(x) \in S$ and $f^{-1}(x) \subset S$. It follows from the definitions that both the Julia and quasi-Fatou sets are completely invariant. Note that it follows from [10, Theorem 1.2] that if $f$ is a transcendental entire function, then $Q F(f)$ is simply the usual Fatou set.

If $G \subset \mathbb{R}^{d}$ is a domain, then we denote by $T(G)$ the topological hull of the domain, in other words the union of $G$ with its bounded complementary components. Note that unlike, for example, in [7], we allow $G$ to be unbounded in this definition. It is well known that if $G \subset \mathbb{R}^{d}$ is a domain, then $T(G)$ is also a domain; see, for example [18, Exercise 2.13.6(c)]. If $G=T(G)$, then we say that $G$ is full. Observe that a domain in the complex plane is full if and only if it is simply connected. If $G \neq T(G)$, then we say that $G$ is hollow.

Our first result is a generalisation of Baker's result in [1].

Theorem 1.1. For each $d \geq 2$, there exists a quasiregular map of transcendental type $f: \mathbb{R}^{d} \rightarrow \mathbb{R}^{d}$ such that $Q F(f)$ has a component which is hollow.

Remark 1. If $\mathbb{R}^{d}=\mathbb{R}^{2}$, which we can identify with $\mathbb{C}$, we can take $f$ to be a transcendental entire function with a multiply connected Fatou component. Our construction in dimensions greater than two is an analogue of Baker's original construction, applied using the functions in [12].

Remark 2. When Baker constructed a multiply connected Fatou component in [1] he did not know if it was bounded. In fact, it was over ten years before he showed [2] that this is indeed the case. He later showed [3] that, in fact, this is always the case for a transcendental entire function. We do not know, however, if the hollow quasi-Fatou component of the function in Theorem 1.1 is bounded or not. It is an open question whether there exists a quasi-Fatou component that is both hollow and unbounded.

We prove two results, which concern the cases that a hollow quasi-Fatou component is either bounded or unbounded. The first shows that quasi-Fatou components of quasiregular maps of transcendental type that are both bounded and hollow have properties very similar to those of multiply connected Fatou components of transcendental entire functions. In order to state this result we need to give a number of definitions, all of which are familiar from complex dynamics.

If $U_{0}$ is a quasi-Fatou component of $f$, then we let $U_{k}$ be the quasi-Fatou component of $f$ containing $f^{k}\left(U_{0}\right)$, for $k \in \mathbb{N}$. (Note that, even for entire $f$, we cannot assume that $U_{k}=f^{k}\left(U_{0}\right)$, although this equality can hold; see Lemma 5.1 below and [19, Lemma 4.1]). If $U_{n} \neq U_{m}$, for $n \neq m$, then we say that $U_{0}$ is wandering.

The escaping set

$$
I(f)=\left\{x: f^{k}(x) \rightarrow \infty \text { as } k \rightarrow \infty\right\}
$$

was first studied for a general transcendental entire function in [13], and plays an important role in complex dynamics. This set was first studied for a quasiregular map in [8]. 
We also use two subsets of the escaping set which themselves now have an important role in complex dynamics. The fast escaping set $A(f)$ can be defined by

(1.2) $A(f)=\left\{x\right.$ : there exists $\ell \in \mathbb{N}$ such that $\left|f^{k+\ell}(x)\right| \geq M^{k}(R, f)$, for $\left.k \in \mathbb{N}\right\}$.

Here $M(R, f)$ denotes the maximum modulus function

$$
M(R, f)=\max _{|x|=R}|f(x)|, \quad \text { for } R>0,
$$

$M^{k}(R, f)$ denotes the $k$ th iterate of $M(R, f)$ with respect to the first variable, and $R>0$ can be taken to be any value such that $M^{k}(R, f) \rightarrow \infty$ as $k \rightarrow \infty$. For a transcendental entire function this form of the definition of $A(f)$ was first used in [21]. This definition was first used for a quasiregular map of transcendental type in [7], where it was shown to be independent of $R$, and equivalent to two other definitions.

We also define the set

$$
A_{R}(f)=\left\{x:\left|f^{k}(x)\right| \geq M^{k}(R, f), \text { for } k \in \mathbb{N}\right\} .
$$

It is known that for a transcendental entire function $f$, the sets $A_{R}(f), A(f)$ and $I(f)$ can have a structure known as a spider's web. We refer to [21, 22] for more background on this structure, which is defined as follows.

Definition 1.2. A set $E \subset \mathbb{R}^{d}$ is a spider's web if $E$ is connected, and there exists a sequence $\left(G_{n}\right)_{n \in \mathbb{N}}$ of bounded full domains with $G_{n} \subset G_{n+1}$, for $n \in \mathbb{N}, \partial G_{n} \subset E$, for $n \in \mathbb{N}$, and $\cup_{n \in \mathbb{N}} G_{n}=\mathbb{R}^{d}$.

We are now able to state our result. Here we denote the Euclidean distance from a point $x$ to a set $U \subset \mathbb{R}^{d}$ by $\operatorname{dist}(x, U)=\inf _{y \in U}|x-y|$. We say that a set $U \subset \mathbb{R}^{d}$ surrounds a set $V \subset \mathbb{R}^{d}$ if $V$ is contained in a bounded component of the complement of $U$.

Theorem 1.3. Suppose that $f: \mathbb{R}^{d} \rightarrow \mathbb{R}^{d}$ is a quasiregular function of transcendental type. Suppose that $U_{0}$ is a quasi-Fatou component of $f$ which is bounded and hollow. Let $R>0$ be such that $M^{k}(R, f) \rightarrow \infty$ as $k \rightarrow \infty$. Then:

(a) each $U_{k}$ is bounded and hollow, $U_{k+1}$ surrounds $U_{k}$ for all large $k$, and also $\operatorname{dist}\left(0, U_{k}\right) \rightarrow \infty$ as $k \rightarrow \infty$;

(b) there exists $\ell \in \mathbb{N}$ such that $\bar{U}_{\ell} \subset A_{R}(f)$, and so $\bar{U}_{0} \subset A(f)$;

(c) the sets $A_{R}(f), A(f)$ and $I(f)$ are spiders' webs.

Remark 3. If $f$ is a transcendental entire function and $U_{0}$ is a Fatou component of $f$, then the assumption that $U_{0}$ is bounded is not required. In this case part (a) is a result of Baker [3, Theorem 3.1], and part (b) and part (c) are due to Rippon and Stallard [21, Theorem 4.4 and Corollary 6.1]. We note that it follows from part (a) that $U_{0}$ is wandering.

Our second result, which concerns quasi-Fatou components of quasiregular maps of transcendental type which are unbounded and hollow, is as follows.

Theorem 1.4. Suppose that $f: \mathbb{R}^{d} \rightarrow \mathbb{R}^{d}$ is a quasiregular function of transcendental type. Suppose that $U$ is a quasi-Fatou component of $f$ which is unbounded and hollow. Then $U$ has no unbounded boundary components and is completely invariant, and all other quasi-Fatou components of $f$ are full. 
Remark 4. If $f$ is a transcendental entire function and $U$ is a Fatou component of $f$, then this result is due to Töpfer [24], although Baker [3, Theorem 3.1] later showed that all multiply connected Fatou components of a transcendental entire function are, in fact, bounded.

The following corollary of Theorem 1.3 and Theorem 1.4 is immediate, and is a generalisation of a well-known fact in complex dynamics.

Corollary 1.5. Suppose that $f: \mathbb{R}^{d} \rightarrow \mathbb{R}^{d}$ is a quasiregular function of transcendental type. If $J(f)$ has a bounded component, then all components of $J(f)$ are bounded.

Finally we consider the implications of two possible configurations of the Julia set of a quasiregular map, $f$, of transcendental type. Firstly, it is known [9, Theorem 1.1] that

$$
J(f) \subset \partial A(f) .
$$

It is also known [9, Theorem 1.2] that if

$$
\liminf _{r \rightarrow \infty} \frac{\log \log M(r, f)}{\log \log r}=\infty
$$

then $J(f)=\partial A(f)$. However, it is not known if it is possible for $Q F(f) \cap \partial A(f)$ to be non-empty. Secondly, it is known that for many quasiregular maps of transcendental type, $J(f)$ is perfect. The paper [10] shows that this holds in a variety of circumstances. It is not known, however, if it is ever possible for $J(f)$ to have an isolated point.

The following theorem considers the case that either $J(f) \neq \partial A(f)$, or that $J(f)$ has an isolated point. Note that if $f$ is a transcendental entire function, then $J(f)=\partial A(f)$ [21, Theorem 5.1] and $J(f)$ is perfect [4, Theorem 3].

Theorem 1.6. Suppose that $f: \mathbb{R}^{d} \rightarrow \mathbb{R}^{d}$ is a quasiregular function of transcendental type. Suppose that $Q F(f) \cap \partial A(f) \neq \emptyset$, or that $J(f)$ has an isolated point. Then $f$ has a quasi-Fatou component which is unbounded and hollow.

A key tool in the proof of these results is the following theorem, which may be of independent interest. Here we denote by $B(a, r)$ the open ball of Euclidean radius $r$, centred at a point $a \in \mathbb{R}^{d}$.

Theorem 1.7. Suppose that $f: \mathbb{R}^{d} \rightarrow \mathbb{R}^{d}$ is a quasiregular function of transcendental type. Let $r>0$ be sufficiently large that $M^{k}(r, f) \rightarrow \infty$ as $k \rightarrow \infty$. Suppose that $G$ is a domain, and that there exist $x, y \in G$ and $\ell_{0}, k_{0} \in \mathbb{N}$ such that

$$
\left|f^{k+\ell_{0}}(y)\right| \leq M^{k-1}(r, f)<M^{k}(r, f) \leq\left|f^{k+\ell_{0}}(x)\right|, \quad \text { for } k \geq k_{0} .
$$

Then there exists $\ell \in \mathbb{N}$ such that

$$
B\left(0, M^{k}(r, f)\right) \subset T\left(f^{k+\ell}(G)\right), \quad \text { for } k \in \mathbb{N} .
$$

Note that the hypothesis (1.4) is satisfied if $G \cap \partial A(f) \neq \emptyset$. We give this slightly more general result in view of potential applications.

The proof of Theorem 1.7 uses a certain conformally invariant metric. Very roughly, the theorem holds since $f$ cannot increase this metric too much, whereas the points $x$ and $y$ must iterate far apart in the Euclidean metric. This can be used to show that the boundary of the topological hull of $f^{k}(G)$ must be far from these 
iterates, for large values of $k$.

The subsequent article [19] contains further results about hollow quasi-Fatou components of quasiregular maps.

The structure of this paper is as follows. First, in Section 2 we recall the definitions of quasiregularity, capacity and the modulus of a curve family, and we give some known results required in the rest of the paper. Next, in Section 3 we prove Theorem 1.1. In Section 4 we prove Theorem 1.7. Finally in Section 5 we prove Theorem 1.3, Theorem 1.4 and Theorem 1.6.

\section{QUASIREgUlAR MAPS, CAPACITY AND THE MODUlus OF A CURVE FAMILY}

We refer to the monographs [20] and [25] for a detailed treatment of quasiregular maps. Here we only recall the definition and the main properties used.

If $d \geq 2, G \subset \mathbb{R}^{d}$ is a domain and $1 \leq p<\infty$, then the Sobolev space $W_{p, l o c}^{1}(G)$ consists of the functions $f: G \rightarrow \mathbb{R}^{d}$ for which all first order weak partial derivatives exist and are locally in $L^{p}$. If $f \in W_{d, l o c}^{1}(G)$ is continuous, then $f$ is quasiregular if there exists a constant $K_{O} \geq 1$ such that

$$
|D f(x)|^{d} \leq K_{O} J_{f}(x) \quad \text { a.e. }
$$

where $D f(x)$ denotes the derivative,

$$
|D f(x)|=\sup _{|h|=1}|D f(x)(h)|
$$

denotes the norm of the derivative, and $J_{f}(x)$ denotes the Jacobian determinant.

We also let

$$
\ell(D f(x))=\inf _{|h|=1}|D f(x)(h)| .
$$

The condition that (2.1) holds for some $K_{O} \geq 1$ is equivalent to the condition that

$$
K_{I} \ell(D f(x))^{d} \geq J_{f}(x) \text { a.e., }
$$

for some $K_{I} \geq 1$. The smallest constants $K_{O}$ and $K_{I}$ such that (2.1) and (2.2) hold are called the outer and inner dilatation of $f$ and are denoted by $K_{O}(f)$ and $K_{I}(f)$. The dilatation of $f$ is denoted by

$$
K(f)=\max \left\{K_{I}(f), K_{O}(f)\right\} .
$$

We say that $f$ is $K$-quasiregular if $K(f) \leq K$, for some $K \geq 1$.

If $f$ and $g$ are quasiregular, with $f$ defined in the range of $g$, then $f \circ g$ is quasiregular and [20, Theorem II.6.8]

$$
K_{I}(f \circ g) \leq K_{I}(f) K_{I}(g) .
$$

Many properties of holomorphic functions extend to quasiregular maps. In particular, we frequently use the fact that non-constant quasiregular maps are open and discrete.

We need a result on the growth of the maximum modulus of a quasiregular map of transcendental type; see [5, Lemma 3.4], [15, Corollary 4.3].

Lemma 2.1. Suppose that $f: \mathbb{R}^{d} \rightarrow \mathbb{R}^{d}$ is a quasiregular function of transcendental type. Then

$$
\lim _{r \rightarrow \infty} \frac{\log M(r, f)}{\log r}=\infty .
$$


Lemma 2.1 has the following corollary, which is immediate.

Corollary 2.2. Suppose that $f: \mathbb{R}^{d} \rightarrow \mathbb{R}^{d}$ is a quasiregular function of transcendental type. If $r>0$ is sufficiently large that $M^{k}(r, f) \rightarrow \infty$ as $k \rightarrow \infty$, then

$$
\lim _{k \rightarrow \infty} \frac{\log \log M^{k}(r, f)}{k}=\infty .
$$

An important tool in the study of quasiregular mappings is the capacity of a condenser, and we recall this concept briefly. Suppose that $A \subset \mathbb{R}^{d}$ is open, and $C \subset A$ is non-empty and compact. The pair $(A, C)$ is called a condenser, and its capacity, which we denote by $\operatorname{cap}(A, C)$, is defined by

$$
\operatorname{cap}(A, C)=\inf _{u} \int_{A}|\nabla u|^{d} d m,
$$

where the infimum is taken over all non-negative functions $u \in C_{0}^{\infty}(A)$ which satisfy $u(x) \geq 1$, for $x \in C$.

If $C$ is compact and $\operatorname{cap}(A, C)=0$ for some bounded open set $A$ containing $C$, then $\operatorname{cap}\left(A^{\prime}, C\right)=0$ for every bounded open set $A^{\prime}$ containing $C$; see [20, Lemma III.2.2]. In this case we write cap $C=0$. Otherwise we write cap $C>0$. For an unbounded closed set $C \subset \mathbb{R}^{d}$, we write cap $C=0$ if cap $C^{\prime}=0$ for every compact subset $C^{\prime}$ of $C$.

If $C$ is closed and cap $C=0$, then $C$ is, in a sense, a small set. In particular we use the fact [20, VII.1.15] that the Hausdorff dimension of $C$ is zero. In particular, if $C$ has an interior point, then cap $C>0$.

A second important tool in the study of quasiregular maps is the concept of the modulus of a curve family; we refer to [20, Chapter II] and [25, Chapter 2] for a detailed discussion. Suppose that $\Gamma$ is a family of paths in $\mathbb{R}^{d}$. A non-negative Borel function $\rho: \mathbb{R}^{d} \rightarrow \mathbb{R} \cup\{\infty\}$ is called admissible if $\int_{\gamma} \rho d s \geq 1$, for all locally rectifiable paths $\gamma \in \Gamma$. We let $\mathcal{F}(\Gamma)$ be the family of all admissible Borel functions, and let the modulus of $\Gamma$ be given by

$$
M(\Gamma)=\inf _{\rho \in \mathcal{F}(\Gamma)} \int_{\mathbb{R}^{d}} \rho^{d} d m .
$$

Suppose that $G \subset \mathbb{R}^{d}$ is a domain, and $E, F$ are subsets of $\bar{G}$. We denote by $\Delta(E, F ; G)$ the family of all paths which have one endpoint in $E$, one endpoint in $F$, and which otherwise are in $G$.

A connection between the capacity of a condenser and the modulus of a path family is the fact [20, Proposition II.10.2] that if $E$ is a compact subset of $G$, then

$$
\operatorname{cap}(G, E)=M(\Delta(E, \partial G ; G)) .
$$

Next we introduce a conformal invariant which is a useful alternative to the hyperbolic metric when working with quasiregular maps. Let $G \subset \mathbb{R}^{d}$ be a domain, and define a function $\mu_{G}$ by

$$
\mu_{G}(x, y)=\inf _{C_{x y}} M\left(\Delta\left(C_{x y}, \partial G ; G\right)\right), \quad \text { for } x, y \in G,
$$

where the infimum is taken over curves $C_{x y}$ which are contained in $G$ and join $x$ and $y$; see [25, p.103] for this definition and more background. It is known that 
$\mu_{G}(x, y)$ is a conformal invariant, and is a metric if cap $\partial G>0$. It is noted in [25] that if $D \subset G$ is a domain, then

$$
\mu_{D}(x, y) \geq \mu_{G}(x, y), \quad \text { for } x, y \in D .
$$

The "transformation formula" for $\mu_{G}$ is as follows [25, Theorem 10.18].

Lemma 2.3. Suppose that $f: G \rightarrow \mathbb{R}^{d}$ is a non-constant quasiregular mapping. Then

$$
\mu_{f(G)}(f(a), f(b)) \leq K_{I}(f) \mu_{G}(a, b), \quad \text { for } a, b \in G .
$$

We require the following estimate for $\mu_{G}$ [25, Theorem 8.31]. (Here we write $\partial_{\infty} G$ for the boundary of $G$ taken in $\overline{\mathbb{R}}^{d}$.)

Lemma 2.4. Suppose that $G$ is a proper subdomain of $\mathbb{R}^{d}$ such that $\partial_{\infty} G$ is connected. Then there exists a constant $c_{d}$, depending only on $d$, such that

$$
\mu_{G}(a, b) \geq c_{d} \log \left(1+\frac{|a-b|}{\min \{\operatorname{dist}(a, \partial G), \operatorname{dist}(b, \partial G)\}}\right), \quad \text { for } a, b \in G .
$$

\section{Proof of Theorem 1.1}

We construct our function using a theorem of Drasin and Sastry in [12]. We need to introduce some terminology before we can state the result we use from that paper.

Suppose that $\nu: \mathbb{R} \rightarrow \mathbb{R}$ is continuous, positive and increasing, and that $\nu(r) \rightarrow \infty$ as $r \rightarrow \infty$. Let $n_{0}$ be an integer greater than $\nu(0)$, and define a sequence of integers $\left(r_{n}\right)_{n \geq n_{0}}$ by

$$
r_{n}=\max \{r: \nu(r)=n\} .
$$

In $[12]$ it is assumed that

$$
r \nu^{\prime}(r)<\nu(r) / 2 \text { and } r \nu^{\prime}(r)=o(\nu(r)) \text { as } r \rightarrow \infty .
$$

In fact (3.2) is only used in [12] to deduce that

$$
n \log \frac{r_{n+1}}{r_{n}} \rightarrow \infty \text { as } n \rightarrow \infty \text {. }
$$

In our case it is somewhat easier to check (3.3) directly, and so we use this equation in the statement of Drasin and Sastry's theorem.

It is helpful to write down a formula for an exceptional set. For $\epsilon \in(0,1)$, we define a union of closed intervals

$$
E_{\epsilon}=\bigcup_{n>n_{0}}\left[\epsilon r_{n}, r_{n}\right]
$$

Define a function

$$
L(r)=\exp \left(\int_{1}^{r} \frac{\nu(t)}{t} d t\right) .
$$

Finally, it is helpful to work with the maximum norm, which is defined by

$$
\|x\|_{\infty}=\max \left\{\left|x_{1}\right|, \ldots,\left|x_{d}\right|\right\}, \quad \text { for } x=\left(x_{1}, \ldots, x_{d}\right) \in \mathbb{R}^{d} .
$$

A statement of Drasin and Sastry's result, which includes part of their construction, is as follows. 
Theorem 3.1. Suppose that $d \geq 2$, that $\nu(r)$ and $L(r)$ are as above, and that (3.3) is satisfied. Then there exist a quasiregular map of transcendental type $f: \mathbb{R}^{d} \rightarrow \mathbb{R}^{d}$, and constants $c, C, R_{0}>0$ and $\epsilon \in(0,1)$ such that

$$
c L\left(\|x\|_{\infty}\right)<\|f(x)\|_{\infty}<C L\left(\|x\|_{\infty}\right), \quad \text { for }\|x\|_{\infty} \in\left[R_{0}, \infty\right) \backslash E_{\epsilon} .
$$

The form of the exceptional set $E_{\epsilon}$ in the statement of Theorem 3.1, which is not made explicit in [12], can be obtained as follows. First, we can deduce from [12, Equation (2.10)], together with the paragraph preceding it, that there exists $\epsilon \in(0,1)$ such that if $\epsilon r_{n+1}>r_{n}$, then the interval $\left[r_{n}, \epsilon r_{n+1}\right]$ lies in the interval labelled $J_{n}^{0}$ by Drasin and Sastry, for all sufficiently large values of $n$. Second, the fact that (3.5) holds when $\|x\|_{\infty} \in J_{n}^{0}$ follows from [12, Equation (3.6)].

Proof of Theorem 1.1. Roughly speaking, we construct a quasiregular function $f$ of transcendental type with the following properties:

(a) the function $f$ behaves like a power map in very large "square rings";

(b) subrings of these can be defined in such a way that $f$ maps these subrings into each other.

Note that this is, essentially, the same idea as Baker's original construction of a multiply connected Fatou component of a transcendental entire function using an infinite product. Properties (a) and (b) are achieved iteratively: if $r_{n}$ is defined, then $\nu$ and $r_{n+1}$ are chosen so that $r_{n+1}$ is much larger than $r_{n}$ (to satisfy (a)), and so that if $\|x\|_{\infty}=r_{n}$, then $\|f(x)\|_{\infty}$ is approximately equal to $r_{n+1}$ (which leads to (b)). Property (b) then ensures that these subrings lie in $Q F(f)$, and the theorem follows.

We now give the full detail of the proof. Let $d \geq 2$ be an integer. We first construct a function $\nu$ with certain properties. We then invoke Theorem 3.1 to obtain a quasiregular map $f: \mathbb{R}^{d} \rightarrow \mathbb{R}^{d}$ such that (3.5) is satisfied. Finally we prove that this function has a hollow quasi-Fatou component.

The construction of $\nu$ is as follows. First choose an integer $n_{0} \geq 2$ and a real number $R^{\prime}>4$. Set $\nu(r)=n_{0}$, for $0 \leq r \leq R^{\prime}$. Since we shall ensure that $\nu(r)>n_{0}$, for $r>R^{\prime}$, this implies that $r_{n_{0}}=R^{\prime}$.

We complete the definition of $\nu$, and the sequence $\left(r_{n}\right)_{n>n_{0}}$ iteratively. Suppose that $n \geq n_{0}$, that $r_{n}$ has been defined, and that $\nu(r)$ has been defined for $0 \leq r \leq r_{n}$. We set

$$
r_{n+1}=L\left(r_{n}\right),
$$

and let $\nu(r)$ be linear; in other words, we set

$$
\nu(r)=\frac{r-r_{n}}{r_{n+1}-r_{n}}+n, \quad \text { for } r_{n} \leq r \leq r_{n+1} .
$$

Note that if $n \geq n_{0}$, then

$$
r_{n+1}=L\left(r_{n}\right)=\exp \left(\int_{1}^{r_{n}} \frac{\nu(t)}{t} d t\right) \geq \exp \left(\int_{1}^{r_{n}} \frac{n_{0}}{t} d t\right) \geq r_{n}^{n_{0}}
$$

It follows from the choices of $n_{0}$ and $R^{\prime}$ that $\nu$ is positive, continuous and increasing, tends to infinity, and that (3.3) holds. Hence we can let $f: \mathbb{R}^{d} \rightarrow \mathbb{R}^{d}$ and constants $c, C, R_{0}>0$ and $\epsilon \in(0,1)$ be as in Theorem 3.1. 
We complete the proof of the theorem by showing that $f$ has a hollow quasi-Fatou component. For each sufficiently large integer $n \geq n_{0}$, define a "square ring"

$$
A_{n}=\left\{x: 2 r_{n}<\|x\|_{\infty}<\epsilon r_{n+1}\right\} .
$$

We claim that for all sufficiently large values of $n$, we have that $f\left(A_{n}\right) \subset A_{n+1}$. It then follows from (1.1) that $A_{n} \subset Q F(f)$. It is easy to see that the theorem follows from this fact, since the Julia set of $f$ is not empty.

Choose $N_{0} \geq n_{0}$ sufficiently large that, for $n \geq N_{0}$ we have that $2 r_{n}<\epsilon r_{n+1}$, that $c 2^{n} \geq 2$, and that $C \epsilon^{n} \leq \epsilon$.

Suppose that $n \geq N_{0}$, and that $x \in A_{n}$. Then, by (3.5), (3.6), (3.7) and since $L(r)$ is increasing

$$
\begin{aligned}
\|f(x)\|_{\infty} & \geq c L\left(2 r_{n}\right) \\
& =c \exp \left(\int_{1}^{r_{n}} \frac{\nu(t)}{t} d t\right) \exp \left(\int_{r_{n}}^{2 r_{n}} \frac{\nu(t)}{t} d t\right) \\
& \geq c L\left(r_{n}\right) \exp \left(\int_{r_{n}}^{2 r_{n}} \frac{n}{t} d t\right) \\
& =c 2^{n} L\left(r_{n}\right) \\
& \geq 2 L\left(r_{n}\right)=2 r_{n+1} .
\end{aligned}
$$

For the same reasons,

$$
\begin{aligned}
\|f(x)\|_{\infty} & \leq C L\left(\epsilon r_{n+1}\right) \\
& =C \exp \left(\int_{1}^{r_{n+1}} \frac{\nu(t)}{t} d t\right) \exp \left(-\int_{\epsilon r_{n+1}}^{r_{n+1}} \frac{\nu(t)}{t} d t\right) \\
& \leq C L\left(r_{n+1}\right) \exp \left(-\int_{\epsilon r_{n+1}}^{r_{n+1}} \frac{n}{t} d t\right) \\
& =\epsilon^{n} C L\left(r_{n+1}\right) \leq \epsilon L\left(r_{n+1}\right)=\epsilon r_{n+2} .
\end{aligned}
$$

Hence $f(x) \in A_{n+1}$, and this completes the proof.

Remark 5. For the function $f$ constructed above, we do not know if $J(f)=\partial A(f)$. The growth of $f$ is too slow to use the result [9, Theorem 1.2] mentioned in the introduction. In fact, it follows from (3.8) that

$$
\log r_{n+n_{0}} \geq n_{0}^{n} \log r_{n_{0}}, \quad \text { for } n \in \mathbb{N} .
$$

We deduce, by (3.4) and (3.5), that

$$
\begin{aligned}
\liminf _{r \rightarrow \infty} \frac{\log \log M(r, f)}{\log \log r} & \leq \liminf _{n \rightarrow \infty} \frac{\log \log M\left(2 r_{n}, f\right)}{\log \log 2 r_{n}} \\
& \leq \liminf _{n \rightarrow \infty} \frac{\log \log \left(C \sqrt{d} L\left(2 r_{n}\right)\right)}{\log \log 2 r_{n}} \\
& \leq \liminf _{n \rightarrow \infty} \frac{\log \log \left(C \sqrt{d}\left(2 r_{n}\right)^{n+1}\right)}{\log \log 2 r_{n}} \\
& \leq \liminf _{n \rightarrow \infty} \frac{\log 2(n+1)+\log \log 2 r_{n}}{\log \log 2 r_{n}}=1 .
\end{aligned}
$$

It does not seem possible to modify the construction to obtain sufficiently fast growth to apply the result of [9, Theorem 1.2], and we currently have no example of a 
quasiregular map with a hollow quasi-Fatou component which does have sufficiently fast growth.

\section{Proof of Theorem 1.7}

In general, if $H$ is a domain, then we let the outer boundary of $H$ be denoted by $\partial_{\text {out }} H=\partial T(H)$.

Suppose that $f: \mathbb{R}^{d} \rightarrow \mathbb{R}^{d}$ is a quasiregular function of transcendental type, and that $G$ is a domain. We can assume we have $T\left(f^{k}(G)\right) \neq \mathbb{R}^{d}$, for $k \in \mathbb{N}$, as otherwise there is nothing to prove.

Let $x, y \in G, r>0$ and $\ell_{0}, k_{0} \in \mathbb{N}$ be as in the statement of the theorem. By Lemma 2.1 and (1.4), we can assume that

$$
2\left|f^{k}(y)\right| \leq\left|f^{k}(x)\right|, \quad \text { for all sufficiently large } k .
$$

We claim that there exists $k_{2} \in \mathbb{N}$ such that

$$
\left.\operatorname{dist}\left(0, \partial_{\text {out }} f^{k+\ell_{0}}(G)\right) \geq M^{k-1}(r, f)\right), \quad \text { for } k \geq k_{2} .
$$

Since $\left|f^{k+\ell_{0}}(y)\right| \leq M^{k-1}(r, f)$, for all $k \geq k_{0}$, it is easy to see that this claim implies that

$$
B\left(0, M^{k}(r, f)\right) \subset B\left(0, M^{k+k_{2}-1}(r, f)\right) \subset T\left(f^{k+k_{2}+\ell_{0}}(G)\right), \quad \text { for } k \in \mathbb{N},
$$

from which Theorem 1.7 follows with $\ell=k_{2}+\ell_{0}$.

To prove (4.2), we first deduce from Lemma 2.3, Lemma 2.4, (2.3), (2.4) and (4.1) that, for all large $k$,

$$
\begin{aligned}
K_{I}(f)^{k} \mu_{G}(x, y) & \geq K_{I}\left(f^{k}\right) \mu_{G}(x, y) \\
& \geq \mu_{f^{k}(G)}\left(f^{k}(x), f^{k}(y)\right) \\
& \geq \mu_{T\left(f^{k}(G)\right)}\left(f^{k}(x), f^{k}(y)\right) \\
& \geq c_{d} \log \left(1+\frac{\left|f^{k}(x)-f^{k}(y)\right|}{\operatorname{dist}\left(f^{k}(y), \partial_{\text {out }} f^{k}(G)\right)}\right) \\
& \geq c_{d}\left(\log \left(\left|f^{k}(x)\right|\right)-\log \left(\operatorname{dist}\left(f^{k}(y), \partial_{\text {out }} f^{k}(G)\right)\right)-\log 2\right) .
\end{aligned}
$$

We deduce by (1.4) that there exist $c>K_{I}(f)$ and $k_{1} \geq k_{0}$ such that

$$
\begin{aligned}
\log M^{k}(r, f) & \leq \log \left|f^{k+\ell_{0}}(x)\right| \\
& \leq c^{k}+\log \left(\operatorname{dist}\left(f^{k+\ell_{0}}(y), \partial_{\text {out }} f^{k+\ell_{0}}(G)\right)\right) \\
& \leq c^{k}+\log \left(\operatorname{dist}\left(0, \partial_{\text {out }} f^{k+\ell_{0}}(G)\right)+\left|f^{k+\ell_{0}}(y)\right|\right), \\
& \leq c^{k}+\log \left(\operatorname{dist}\left(0, \partial_{\text {out }} f^{k+\ell_{0}}(G)\right)+M^{k-1}(r, f)\right), \quad \text { for } k \geq k_{1} .
\end{aligned}
$$

By Lemma 2.1 and Corollary 2.2 , we can choose $k_{2} \geq k_{1}$ sufficiently large that all the following hold for $k \geq k_{2}$ :

(i) $\log \log M^{k-1}(r, f) \geq 2 k \log c$;

(ii) $\log M^{k}(r, f) \geq 2 \log M^{k-1}(r, f)$;

(iii) $c^{2 k} \geq c^{k}+\log 2$. 
We deduce by (4.3) that, for $k \geq k_{2}$,

$$
\begin{aligned}
c^{k}+\log \left(\operatorname{dist}\left(0, \partial_{\text {out }} f^{k+\ell_{0}}(G)\right)+M^{k-1}(r, f)\right) & \geq \log M^{k}(r, f) \\
& \geq 2 \log M^{k-1}(r, f) \\
& \geq \log M^{k-1}(r, f)+c^{2 k} \\
& \geq \log M^{k-1}(r, f)+c^{k}+\log 2 .
\end{aligned}
$$

Equation (4.2) follows, and this completes the proof.

\section{Proofs of Theorem 1.3, Theorem 1.4 And Theorem 1.6}

We require the following topological lemma.

Lemma 5.1. Suppose that $f: \mathbb{R}^{d} \rightarrow \mathbb{R}^{d}$ is a quasiregular function, and that $U$ is a quasi-Fatou component of $f$ which is bounded and full. Then $f(U)$ is bounded and full, and the quasi-Fatou component of $f$ containing $f(U)$ is equal to $f(U)$.

Proof. Suppose that $U$ is a quasi-Fatou component of $f$ which is bounded and full. Clearly $f(U)$ is bounded. We claim that $\partial f(U)$ is connected. For, since $f$ is an open map and $U$ is bounded, $\partial f(U) \subset f(\partial U)$. Moreover, since $f$ is continuous we have

$$
f(\partial U) \subset f(\bar{U}) \subset \overline{f(U)} .
$$

Since $f(\partial U) \subset J(f)$ and $f(U) \subset Q F(f)$, it follows from (5.1) that

$$
f(\partial U) \subset \overline{f(U)} \backslash f(U)=\partial f(U) .
$$

We deduce that $f(\partial U)=\partial f(U)$. Since $U$ is bounded and full, $\mathbb{R}^{d} \backslash U$ is connected and so $\partial U$ is connected; see [14, Theorem 1.6(3)] or [16, $\S 57, \mathrm{I}-\mathrm{II}]$. Hence $\partial f(U)$ is connected, as claimed.

Since $f(U)$ is bounded, if it were also hollow, then $\partial f(U)$ would have at least two components. Hence $f(U)$ is full.

Since $\partial f(U)=f(\partial U) \subset J(f)$, we also have that the quasi-Fatou component of $f$ containing $f(U)$ is equal to $f(U)$.

We now prove Theorem 1.3.

Proof of Theorem 1.3. Suppose that $f: \mathbb{R}^{d} \rightarrow \mathbb{R}^{d}$ is a quasiregular function of transcendental type, and that $U_{0}$ is a quasi-Fatou component of $f$ which is bounded and hollow.

Since $T\left(U_{0}\right) \cap J(f) \neq \emptyset$, it follows from (1.3) that $T\left(U_{0}\right) \cap A(f) \neq \emptyset$. Since $U_{0}$ is bounded, it follows by the maximum principle that $U_{0}$ is wandering.

Our proof now splits into two parts. First we show that there exists a bounded complementary component of $U_{0}$ which contains a point of $J(f)$ in its interior. We then use this fact to prove the theorem.

Since $T\left(U_{0}\right) \cap J(f) \neq \emptyset$, it follows from (1.1) that there exist $x \in T\left(U_{0}\right)$ and $k \geq 1$ such that $f^{k}(x) \in U_{0}$. Let $V$ be the quasi-Fatou component of $f$ containing $x$. Then $V \subset T\left(U_{0}\right)$ and, since $U_{0}$ is wandering, we have $V \cap U_{0}=\emptyset$.

Moreover, $V$ is hollow by Lemma 5.1. Hence $T(V)$ contains a point of $J(f)$. Let $E$ be the component of the complement of $U_{0}$ containing $T(V)$. It follows that int $E \cap J(f) \neq \emptyset$, as claimed.

Let $G_{0}=\operatorname{int} E$. It follows by the maximum principle that $f\left(G_{0}\right) \subset T\left(f\left(U_{0}\right)\right)$. Moreover, $\partial G_{0} \subset J(f)$, and so $f\left(\partial G_{0}\right)$ cannot meet $f\left(U_{0}\right)$. It follows that we 
can let $G_{1}$ be the interior of the bounded complementary component of $f\left(U_{0}\right)$ which contains $f\left(G_{0}\right)$. In general, we can let $G_{k}$ be the interior of the bounded complementary component of $f^{k}\left(U_{0}\right)$ which contains $f^{k}\left(G_{0}\right)$, for $k \in \mathbb{N}$. Clearly $G_{k}$ is full, for $k \in \mathbb{N}$.

Suppose that $k \in \mathbb{N}$. Since $\partial G_{0} \subset J(f)$, we have $f^{k}\left(U_{0}\right) \cap f^{k}\left(\partial G_{0}\right)=\emptyset$, and so $f^{k}\left(\partial G_{0}\right) \subset \overline{G_{k}}$. Clearly $f^{k}\left(\partial G_{0}\right) \cap G_{k}=\emptyset$, since otherwise $G_{k}$ contains a point of $f^{k}\left(U_{0}\right)$. Hence $f^{k}\left(\partial G_{0}\right) \subset \partial G_{k}$. Thus, since $f^{k}$ is an open map and $G_{0}$ is bounded,

$$
\partial f^{k}\left(G_{0}\right) \subset f^{k}\left(\partial G_{0}\right) \subset \partial G_{k} .
$$

Since $G_{k}$ is full, we deduce that

$$
f^{k}\left(G_{0}\right)=G_{k}, \quad \text { for } k \in \mathbb{N} .
$$

Let $R>0$ be sufficiently large that $M^{k}(R, f) \rightarrow \infty$ as $k \rightarrow \infty$. Since we have that $G_{0} \cap J(f) \neq \emptyset$, it follows by (1.3) that $G_{0} \cap \partial A(f) \neq \emptyset$, and so we can apply Theorem 1.7 with $G=G_{0}$. We deduce, by Theorem 1.7 and (5.2) that there exists $\ell \in \mathbb{N}$ such that

$$
B\left(0, M^{k}(R, f)\right) \subset T\left(f^{k+\ell}\left(G_{0}\right)\right)=T\left(G_{k+\ell}\right)=G_{k+\ell}, \quad \text { for } k \in \mathbb{N} .
$$

Theorem 1.3 part (a) and part (b) follow.

To prove part (c), we note first that it follows from Theorem 1.3 part (b) and (1.3) that the complement of $A_{R}(f)$ has a bounded component. The fact that the sets $A_{R}(f)$ and $A(f)$ are both spiders' webs follows immediately from [7, Proposition $6.2]$.

It remains to show that $I(f)$ is a spider's web. Since $A(f)$ is a spider's web, and so connected, we can let $I_{0}$ be the component of $I(f)$ which contains $A(f)$. By definition $I_{0}$ is a spider's web. We show that, in fact, $I(f)=I_{0}$.

First, suppose that $x \in I(f) \cap J(f)$. Then $x \in \partial A(f)$, by (1.3), and so $x \in \overline{I_{0}}$. Thus $I_{0} \cup\{x\}$ is connected and, since $x \in I(f)$ and $I_{0}$ is a component of $I(f)$, it follows that $x \in I_{0}$. Thus

$$
(I(f) \cap J(f)) \subset I_{0} .
$$

Next, suppose that $x \in I(f) \cap Q F(f)$. Let $V_{0}$ be the component of $Q F(f)$ containing $x$. We claim that $\overline{V_{0}} \subset I(f)$. It is easy to see that the fact that $I(f)=I_{0}$ follows from this claim, by (5.3).

Recall that $V_{n}$ (resp. $U_{n}$ ) denotes the Fatou component containing $f^{n}\left(V_{0}\right)$ (resp. $\left.f^{n}\left(U_{0}\right)\right)$. If $V_{n}=U_{m}$, for some $n, m \in \mathbb{N}$, then $\overline{V_{n}} \subset A(f)$, by Theorem 1.3 part (b). So we can assume that

$$
V_{n} \neq U_{m}, \quad \text { for } n, m \in \mathbb{N} .
$$

For all sufficiently large values of $n$, let $B_{n}$ denote the intersection of the unbounded complementary component of $U_{n}$ with $T\left(U_{n+1}\right) \backslash U_{n+1}$. Since $f^{n}(x) \rightarrow \infty$, it follows by (5.4) that there is a sequence $\left(k_{n}\right)_{n \in \mathbb{N}}$ such that $f^{n}(x) \in B_{k_{n}}$, for all sufficiently large $n \in \mathbb{N}$, and $k_{n} \rightarrow \infty$ as $n \rightarrow \infty$.

It follows by (5.4) that $\overline{V_{n}} \subset \overline{B_{k_{n}}}$, for all sufficiently large $n \in \mathbb{N}$, and the result follows.

Next we prove Theorem 1.4. 
Proof of Theorem 1.4. Suppose that $f: \mathbb{R}^{d} \rightarrow \mathbb{R}^{d}$ is a quasiregular function of transcendental type. Suppose that $U$ is a quasi-Fatou component of $f$ which is unbounded and hollow.

Let $E$ be a bounded complementary component of $U$. We claim that there is a bounded full domain, $G$, such that $E \subset G$ and $\partial G \subset U$. We prove this claim as follows. For each $n \in \mathbb{N}$, let $V_{n}$ be the component of $\left\{y: \operatorname{dist}\left(y, U^{c}\right) \leq 1 / n\right\}$ that contains $E$.

Suppose that all the sets $V_{n}$ are unbounded. Let $\rho>0$ be sufficiently large that $E \subset B(0, \rho / 2)$. For each $n \in \mathbb{N}$, let $V_{n}^{*}$ be the component of $V_{n} \cap \overline{B(0, \rho)}$ containing $E$. It is easy to see that each $V_{n}^{*}$ is a continuum. Let $V^{*}=\bigcap_{n \in \mathbb{N}} V_{n}^{*}$. Then $V^{*}$ is a nested intersection of continua, and so [17, Theorem 1.8] is a continuum. In particular $V^{*}$ is connected.

Since $U$ is open, $V^{*} \subset U^{c}$. Now $E=V^{*}$, since $E \subset V^{*}$ and $E$ is a component of $U^{c}$. Also, $V^{*}$ contains the non-empty set $\bigcap_{n \in \mathbb{N}}\left(V_{n}^{*} \cap \partial B(0, \rho)\right)$, and so also contains a point of modulus $\rho$. This is a contradiction.

It follows that there exists $n \in \mathbb{N}$ such that $V_{n}$ is bounded. We let $G$ be the topological hull of the component of $\left\{y: \operatorname{dist}\left(y, U^{c}\right)<1 / n\right\}$ that contains $E$. This completes the proof of our claim.

It follows from [7, Proposition 2.4] that

$$
\partial T\left(f^{k}(G)\right) \subset f^{k}(\partial T(G))=f^{k}(\partial G) \subset f^{k}(U) .
$$

Since $E \cap J(f) \neq \emptyset$, we have by $(1.3)$ that $G \cap \partial A(f) \neq \emptyset$. We apply Theorem 1.7 with $r>0$ sufficiently large that $M^{k}(r, f) \rightarrow \infty$ as $k \rightarrow \infty$.

Since $U$ is unbounded, we deduce from (1.5) that $\partial T\left(f^{k}(G)\right) \cap U \neq \emptyset$ for all sufficiently large values of $k$. Hence, by (5.5), $U$ meets $f^{k}(U)$ and $f^{k+1}(U)$, for some $k$, and so $U$ is the quasi-Fatou component containing $f(U)$. We deduce similarly by (1.5) and (5.5) that $U$ has no unbounded boundary components.

Next, suppose that $V \neq U$ is a quasi-Fatou component of $f$ such that $f(V) \subset U$. Since $U$ has no unbounded boundary components, all complementary components of $U$ are bounded, and so $V$ is bounded. It follows by Lemma 5.1 that $V$ is hollow. We deduce a contradiction by Theorem 1.3 part (a). Hence $U$ is completely invariant.

Finally, it is straightforward to see that Theorem 1.3 part (a) implies that $f$ has no other hollow quasi-Fatou components.

We note the following corollary, which is a generalisation of a well-known result for transcendental entire functions.

Corollary 5.2. Suppose that $f: \mathbb{R}^{d} \rightarrow \mathbb{R}^{d}$ is a quasiregular function of transcendental type. Suppose that $U$ is a quasi-Fatou component of $f$, and $V$ is the quasi-Fatou component of $f$ containing $f(U)$. Then $U$ is full if and only if $V$ is full.

Proof. If $U$ is hollow, then $V$ is hollow by Theorem 1.3 and Theorem 1.4. If $U$ is bounded and full, then $V$ is full, by Lemma 5.1. Finally, it is easy to see that if $U$ is unbounded and full, then $f$ has no hollow quasi-Fatou components.

Finally, we prove Theorem 1.6.

Proof of Theorem 1.6. Suppose that $f: \mathbb{R}^{d} \rightarrow \mathbb{R}^{d}$ is a quasiregular function of transcendental type. Suppose first that there exists $x \in Q F(f) \cap \partial A(f)$. Let $G$ be 
a domain containing $x$ and contained in $Q F(f)$. We apply Theorem 1.7 with $r>0$ sufficiently large that $M^{k}(r, f) \rightarrow \infty$ as $k \rightarrow \infty$.

It follows from (1.5), and from the fact that $J(f) \neq \emptyset$, that there exists $k \in \mathbb{N}$ and a hollow quasi-Fatou component $U$ such that $f^{k}(G) \subset U$. Since $G \cap A(f)^{c} \neq \emptyset$, it follows from Theorem 1.3 part (b) that $U$ is unbounded. This completes the proof of the first part of the theorem.

Suppose second that $J(f)$ has an isolated point, $x$. Let $G$ be a domain such that $G \cap J(f)=\{x\}$. By (1.3) we have that $G \cap A(f)^{c} \neq \emptyset$. Clearly $f$ has a hollow quasiFatou component $U$ which contains $G \backslash\{x\}$. Then Theorem 1.3 part (b) implies that $U$ is unbounded.

Acknowledgment: The authors are grateful to the referees for useful comments.

\section{REFERENCES}

[1] BAKer, I. N. Multiply connected domains of normality in iteration theory. Math. Z. 81 (1963), 206-214.

[2] Baker, I. N. An entire function which has wandering domains. J. Austral. Math. Soc. Ser. A 22, 2 (1976), 173-176.

[3] Baker, I. N. Wandering domains in the iteration of entire functions. Proc. London Math. Soc. (3) 49, 3 (1984), 563-576.

[4] Bergweiler, W. Iteration of meromorphic functions. Bull. Amer. Math. Soc. (N.S.) 29, 2 (1993), 151-188.

[5] Bergweiler, W. Fixed points of composite entire and quasiregular maps. Ann. Acad. Sci. Fenn. Math. 31, 2 (2006), 523-540.

[6] Bergweiler, W. Fatou-Julia theory for non-uniformly quasiregular maps. Ergodic Theory Dynam. Systems 33, 1 (2013), 1-23.

[7] Bergweiler, W., Drasin, D., And Fletcher, A. The fast escaping set for quasiregular mappings. Anal. Math. Phys. 4, 1-2 (2014), 83-98.

[8] Bergweiler, W., Fletcher, A., Langley, J., and Meyer, J. The escaping set of a quasiregular mapping. Proc. Amer. Math. Soc. 137, 2 (2009), 641-651.

[9] Bergweiler, W., Fletcher, A., And Nicks, D. A. The Julia set and the fast escaping set of a quasiregular mapping. Comput. Methods Funct. Theory 14, 2-3 (2014), 209-218.

[10] Bergweiler, W., AND Nicks, D. A. Foundations for an iteration theory of entire quasiregular maps. Israel J. Math. 201, 1 (2014), 147-184.

[11] Bergweiler, W., Rippon, P. J., and Stallard, G. M. Multiply connected wandering domains of entire functions. Proc. Lond. Math. Soc. (3) 107, 6 (2013), 1261-1301.

[12] Drasin, D., And Sastry, S. Periodic quasiregular mappings of finite order. Rev. Mat. Iberoamericana 19, 3 (2003), 755-766.

[13] Eremenko, A. E. On the iteration of entire functions. Dynamical systems and ergodic theory (Warsaw 1986) 23 (1989), 339-345.

[14] García-Máynez, A., And Illanes, A. A survey on unicoherence and related properties. An. Inst. Mat. Univ. Nac. Autónoma México 29 (1989), 17-67.

[15] Järvi, P. On the zeros and growth of quasiregular mappings. J. Anal. Math. 82 (2000), $347-362$.

[16] Kuratowski, K. Topology, Vol. II, Translated from the French by A. Kirkor. Academic Press, New York-London; PWN Polish Scientific Publishers, Warsaw, 1968.

[17] NADler, JR., S. B. Continuum theory, vol. 158 of Monographs and Textbooks in Pure and Applied Mathematics. Marcel Dekker, Inc., New York, 1992.

[18] Napier, T., and Ramachandran, M. An introduction to Riemann surfaces. Cornerstones. Birkhäuser/Springer, New York, 2011.

[19] Nicks, D. A., AND Sixsmith, D. J. The size and topology of quasi-Fatou components of quasiregular maps. To appear in Proc. Amer. Math. Soc. arXiv:1601.03308.

[20] Rickman, S. Quasiregular mappings, vol. 26 of Ergebnisse der Mathematik und ihrer Grenzgebiete (3). Springer-Verlag, Berlin, 1993.

[21] Rippon, P. J., and Stallard, G. M. Fast escaping points of entire functions. Proc. London Math. Soc. (3) 105, 4 (2012), 787-820. 
[22] Sixsmith, D. J. Entire functions for which the escaping set is a spider's web. Math. Proc. Cambridge Philos. Soc. 151, 3 (2011), 551-571.

[23] Sixsmith, D. J. On fundamental loops and the fast escaping set. J. Lond. Math. Soc. (2) 88, 3 (2013), 716-736.

[24] Töpfer, H. Über die Iteration der ganzen transzendenten Funktionen, insbesondere von $\sin z$ und $\cos z$. Math. Ann. 117 (1939), 65-84.

[25] Vuorinen, M. Conformal geometry and quasiregular mappings, vol. 1319 of Lecture Notes in Mathematics. Springer-Verlag, Berlin, 1988.

[26] Zheng, J.-H. On multiply-connected Fatou components in iteration of meromorphic functions. J. Math. Anal. Appl. 313, 1 (2006), 24-37.

School of Mathematical Sciences, University of Nottingham, Nottingham NG7 2RD, $\mathrm{UK}$

E-mail address: Dan.Nicks@nottingham.ac.uk

School of Mathematical Sciences, University of Nottingham, Nottingham NG7 2RD, $\mathrm{UK}$

E-mail address: David.Sixsmith@open.ac.uk 